\title{
Setting, Structure, and Timing of the Preparticipation Examination: The Wilderness Adventure Consultation
}

\author{
Gary A. Green, MD \\ From the UCLA Division of Sports Medicine, Pepperdine University, Malibu, California.
}

\begin{abstract}
Patients pursue wilderness experiences throughout the entire life cycle, and while outdoor pursuits are relatively safe, injuries do occur. Many of these adverse events can be anticipated, identified, and prevented through a wilderness preparticipation examination (PPE). To accomplish this, it is incumbent on the physician to assess the extrinsic and intrinsic factors faced by the patient and attempt to correct them to ensure an enjoyable experience in the outdoors. This article outlines the goals of the PPE along with identification of various risk factors that can influence a trip. Most injuries and rescues occur from underestimating the risks from extrinsic, environmental factors, and/or overestimating one's intrinsic skills. By matching the patient's fitness and skill level to the environment, the physician can help reduce the risk of serious injury.
\end{abstract}

Key words: PPE, wilderness, intrinsic factors, extrinsic factors, injury prevention

\section{Introduction}

Failure to prepare is preparing to fail. -John Wooden

The basic skills required to do a complete physical examination are ingrained in physicians from the early years of medical school. Those skills are refined during primary care residencies so that physicians have a great deal of expertise in doing complete physical examinations for such things as annual physicals, school physicals, well-woman checks, well-child checks, and preoperative examinations. Although each of these examinations has different objectives, they share a common theme of being preventative in nature by identifying risk factors for future medical problems. In recent years, primary care sports medicine physicians have expanded this concept to the sports preparticipation examination (PPE). In addition to the identification of general health risks, the PPE assesses the demands of a specific sport and determines if the patient is medically able to participate in that particular

The author reports no conflicts of interest.

This article appears in a "Care of the Wilderness and Adventure Athlete" special issue, jointly published by Clinical Journal of Sport Medicine and Wilderness \& Environmental Medicine.

Corresponding author: Gary A. Green, MD, UCLA Division of Sports Medicine, Pepperdine University, Major League Baseball, Pacific Palisades Medical Group, 15200 Sunset Boulevard, Suite 107, Malibu, Pacific Palisades, CA 90272 (e-mail: ggreen@mednet.ucla.edu). sport. The PPE combines the traditional rigorous understanding of pathophysiology with an appreciation for the physical requirements of various sports.

The wilderness PPE builds on the concepts of the PPE but has an additional requirement of understanding the environmental demands that the patient is entering into. In addition to the environmental aspect of the wilderness PPE, there is another area that separates this type of examination from the traditional sports PPE: patient age. Although the majority of patients who participate in organized sports requiring a sports PPE is limited to the ages 13 to 22 years (except for a small number of professional athletes), wilderness pursuits can be performed throughout the life cycle. A National Forest Service survey from 2005 to 2009 revealed that $41 \%$ of visitors to wilderness areas were between the ages of 40 and 60 years with $3 \%$ above the age of 70 years. ${ }^{1}$ Owing to that, it is probable that every primary care physician will have patients who engage in outdoor pursuits, regardless of their geographic location or patient demographics. In addition to the changing demands of exercise with age, there is also the increased likelihood that older patients will have underlying diseases and medications that influence their ability to exercise and must be taken into account. Fortunately, the injury rates for most activities are relatively low, but injuries do occur, and there are risks that can be anticipated and mitigated. Thus, it is incumbent on all practitioners to become familiar with the basics of a 
wilderness PPE. Defining what types of activities should have a PPE and who should undergo evaluation is covered later in this article.

\section{Methods}

A PubMed search was initiated to identify articles with the key words wilderness PPE. Articles were assessed as to their relevance to injury prevention and outdoor experience.

\section{Goals of the Preparticipation Examination}

One of the similarities to the sports PPE is that there are multiple goals for the wilderness PPE. A common misperception is that the main purpose of the PPE is to disqualify athletes for whom participation represents unacceptable risks. If this were the only goal, then most PPEs would be considered failures. It is estimated that only $3 \%$ to $13 \%$ of those screened in sports PPEs require additional evaluation and the absolute disqualification rate is less than $1 \% .^{2}$ Performing millions of sports PPE every year to discover the $1 \%$ who are disqualified would be a terrible waste of resources if this were the only reason for the examination. However, as a physician may evaluate and discuss many different health issues during a patient visit, there are also multiple goals that can be accomplished during the wilderness PPE, which rarely result in complete disqualification.

Although absolute disqualification is relatively uncommon, it is still important to identify those potentially fatal conditions in the wilderness PPE. The condition most commonly associated with all types of exercise is sudden cardiac death; however, there are many disorders that would preclude certain types of activities, and those will be discussed in this theme issue. Although underlying cardiac disorders such as hypertrophic cardiomyopathy are the most frequent causes of sudden death in young athletes, the majority of those seeking a wilderness PPE is older and would be most at risk for coronary artery disease. ${ }^{3}$

Ruling out risk factors for sudden cardiac death in patients younger than 21 years has been well studied through the traditional PPE for athletes. Questions should include family history of premature sudden death and exertional symptoms such as chest pain and syncope. ${ }^{4}$ For older patients, coronary artery disease is the main concern for sudden death. Fortunately, there are established guidelines to help determine who is at risk and the need for exercise stress testing. ${ }^{5}$ The key points for assessing risk are (1) cardiovascular risk factors, (2) baseline exercise capacity, and (3) anticipated exercise intensity. The major cardiovascular risks are well known and include hypertension, diabetes, adverse lipid profile, family history, and tobacco use.
The simplest method of assessing exercise capacity and planned exercise intensity is to use metabolic equivalents (METS). One metabolic equivalent is defined as the amount of oxygen consumed while sitting at rest and is equal to 3.5 $\mathrm{mL}$ oxygen per kilogram body weight $\times$ minutes. $^{6}$ By knowing a patient's baseline METS and their anticipated wilderness METS, the clinician can determine a patient's fitness relative to their expected demands. Table 1 lists the METS of some common outdoor pursuits. It is worth noting that light activity is considered from 1 to 4 METS, moderate intensity at 5 to 8 METS, and heavy exertion greater than 8 METS. $^{6}$ One limitation of the METS calculations is that these are estimated at sea level. If the patient is doing similar activities at altitude, the energy expenditures can increase dramatically. By understanding a patient's functional baseline capacity, the clinician can determine appropriate training and the need for exercise testing. The American College of Sports Medicine and the American Heart Association have established guidelines for cardiovascular screening that provide an excellent reference. ${ }^{5}$ The key is determining the discrepancy between the actual fitness level of the patient and the expected demands. If the difference is large, then exercise stress testing should be considered along with a graduated training program to help the patient accommodate to the anticipated demands.

A further goal of the wilderness PPE is to satisfy liability requirements. Many types of programs and organized trips require a "medical clearance" by a physician before allowing people to participate in their

Table 1. METS of various activities

\begin{tabular}{lccc}
\hline & \multicolumn{3}{c}{ METS } \\
\cline { 2 - 4 } Activity & Light & Moderate & Heavy \\
\hline Alpine skiing & 4 & 6 & 8 \\
Backpacking at 5\% & 6 & 8 & 10 \\
slope carrying 20 kg, km/h & & & \\
6.4 & 8 & & \\
7.2 & 9.6 & & \\
8 & 11.6 & & \\
9.6 & 13.1 & & \\
11.2 & 15.5 & & \\
Cross country skiing, km/h & & & \\
4 & 5.5 & & \\
6 & 7.7 & & \\
8 & 9.9 & & \\
10 & 12.2 & & 10 \\
12 & 14.3 & & \\
14 & 16.5 & & \\
Mountaineering & 7 & 8 & \\
Orienteering Jogging, km/h & 8 & 10 & \\
9 & 8.8 & & \\
11 & 11.2 & & \\
& & &
\end{tabular}


activities. These range from a simple generic statement of health to a complex examination requiring specific testing. Although the physician should of course satisfy the requirements of the form, he/ she should also use the opportunity to explore other goals of the wilderness PPE as outlined in this article. Many of these forms target minimizing liability for outfitters and vendors and should not be used as a definitive guide to the evaluation of patient to ensure safe and optimal participation.

The wilderness PPE can be used to help reduce the risk of injuries in outdoor activities; however, injury reduction is a difficult proposition for several reasons. The first is that the actual injury rate in outdoor pursuits is problematic because of a lack of a quantifiable accurate denominator. For example, is everyone who enters a national park at risk for injury? Under this scenario, the actual injury rate would be quite low because there are many people who drive through a national park and rarely leave their cars. It would be inaccurate to lump those people with someone rock climbing in the same park. As such, demonstrating a reduction in injuries is difficult without accurate denominators. The best data are from controlled programs, such as the National Outdoor Leadership School, in which participants are fairly homogenous with respect to their activities. National Outdoor Leadership School found that injuries occurred at a rate of 1.18 per thousand program days and illness at 1.08 per thousand program days. ${ }^{7}$ The authors found that many of the injuries could have been prevented by reducing pack weights, emphasizing warm-up and stretching, gaining prior experience with uneven terrain, proper recognition, and correction of preexisting injuries. The latter point has been repeatedly observed in sports medicine, and it is fairly obvious that past injuries predispose patients to repeated injury.

Owing to the difficulty in establishing a true denominator for activity, injury reduction is difficult to quantify. However, another end point that has been studied is emergency rescues. A 10-year study of Yosemite rescues found that the most common reasons for rescue were lower extremity injuries, dehydration, hypovolemia, and hunger. ${ }^{8}$ Clearly the latter 3 are all potentially preventable and could be addressed in the wilderness PPE. A study of hiker habits in the White Mountains of New Hampshire concluded that rescues could be reduced by better hiker preparedness. ${ }^{9}$ They also found that short hikes were deemed less risky by hikers, and they were less prepared. The authors determined that the most underprepared groups tended to be younger, less fit, and inexperienced. Again, identifying fitness and experience in the wilderness PPE and helping the patient correct these before an outdoor experience would be expected to help reduce rescues.

By identifying risk factors, the physician can reduce the injuries, illness, and potential rescues and hopefully enhance the outdoor experience for patients. Through knowledge of the anticipated demands, the physician can counsel in preparedness and help construct a training program that will meet the needs of the patient and allow for an optimal experience. In many cases, the physician may need to suggest the use of a coach, trainer, sportspecific instructor, or expedition guide to assist the patient in their preparation.

\section{Extrinsic Versus Intrinsic Risk Factors}

To reduce injuries, illnesses, and rescues through the wilderness PPE, it is imperative to correctly identify risk factors. These factors can be divided into extrinsic and intrinsic types (Tables 2 and 3)..$^{10}$ This grouping system will be recognizable to sports medicine physicians as the concept is similar to the evaluation of sports overuse injuries. It is imperative that the physician be very thorough in uncovering as many of these risks as possible when evaluating a patient for a wilderness experience.

Extrinsic factors (Table 2) are those environmental demands that can potentially affect the patient during his/ her outdoor pursuits and can also be referred to as objective risks. The physician (and patient!) must know as much as possible about the anticipated travel to identify these factors. It is not enough to know where the patient is planning on traveling, but time of year and even time of the day are important variables. For example, in some terrains (eg, the desert), there can be 30 to $40^{\circ} \mathrm{F}$ temperature changes between early morning and noon. The more information about the trip that the patient can obtain before the PPE, the better able to prepare for all eventualities and decrease risk.

Patients who may not be familiar with outdoor pursuits may frequently underestimate these extrinsic factors. For example, equipment is a significant extrinsic factor that is often overlooked by the novice. A blister from new shoes is a minor problem in the city but becomes a major issue and can ruin an experience if an individual has to walk a significant distance in the

Table 2. Extrinsic factors-environmental demands that affect the patient

Temperature
Terrain
Altitude
Weather
Infection risk, for example, insects, parasites, contaminated
wounds
Dangerous wildlife and plants
Demands in terms of METS
Available resources and ease of evacuation


Table 3. Intrinsic factors-factors that the patient brings to the environment

\begin{tabular}{l}
\hline Wilderness experience \\
General experience \\
Experience on particular conditions, specific place \\
Age \\
Physical fitness level \\
Psychological well-being \\
Specific skill level \\
Previous injuries \\
Underlying medical conditions \\
Immunizations \\
Medications
\end{tabular}

wilderness. One of many errors in an infamous fatal Everest expedition was that several of the novice hikers used their hiking boots for the first time at the start of the trip. ${ }^{11}$ Blisters not only can ruin a trip for the person but can affect an entire group. Although unanticipated hazards can certainly present themselves, almost all extrinsic factors can be attenuated through proper preparation. One of the common themes of wilderness rescues is that participants underestimated the risks of extrinsic factors.

The other category is intrinsic factors (Table 3), which are features that the patient brings with him/her to the outdoor environment and are also referred to as subjective risks. As with the extrinsic factors, the physician needs to do a thorough history and physical examination to discover these limitations. Given enough lead time, these factors are generally correctable and can lead to a more enjoyable experience. The problem often arises when a patient presents a short time before the anticipated trip, and there is inadequate preparation time to match the demands presented by the extrinsic factors. Again, the sports medicine overuse analogy is useful in this situation. One would no more recommend an inexperienced individual going on a 30-mile 3-day backpacking trip carrying a 50-pound pack than suggesting someone who rarely runs doing a marathon without training. Although one could certainly survive backpacking without proper preparation, the likelihood for injury and a poor experience is high and would not be advisable. Either altering the trip or correcting the deficiency can correct many of these issues. As an example, a graduated increase in pack weight over a 6-week training period can help prepare for a backpacking trip, as can the suggestion of using hiking poles to reduce hip, back, and knee pain.

Intrinsic factors can affect the ability to participate in wilderness activities. Some of the more significant factors are prior injuries, physical and mental fitness, underlying medical conditions, and medications. As stated above, prior injuries are the best predictors of future injuries, especially if not completely rehabilitated. For example, a relatively minor muscle strain can become a major issue when carrying a heavy pack over several hours going uphill. General physical fitness level is relatively easy to assess as mentioned above with estimated METS. In contrast, psychological wellbeing is harder to assess yet may affect survival in wilderness situations as much or more than physical fitness. Assessing the ability to function under stress may be a significant risk factor in certain experiences.

Many medical diseases, such as asthma and chronic obstructive pulmonary disease will affect the ability to adequately oxygenate at higher altitudes. Even relatively minor conditions such as Raynaud syndrome can make life miserable for a patient in cold environments unless proper precautions are taken and can even threaten fingers and toes. Finally, there are many medications that can affect performance in outdoor pursuits. For example, diuretics can lead to dehydration, certain medications interfere with acclimatization, and there are many medications that cause sun sensitivity. The interaction of medications with altitude, temperature, and environmental factors should be considered. Medications can also pose a risk if they are forgotten, lost, or exposed to the elements, so the patient should be counseled on this as well.

Although many of the intrinsic factors are familiar to most clinicians, wilderness experience is a less familiar quality. As previously cited, many rescues are the result of ill-prepared novice hikers who do not have adequate outdoor experience to handle hazards. One of the key questions in the wilderness PPE is to inquire about previous outdoor experience. However, this must be taken a step further to inquire about specific experience in the anticipated terrain and environment. A person may have a great deal of expertise in a certain geographical area but be ignorant of risk factors elsewhere. "Local knowledge" is a key component of any successful trip, and the more information one can gather before a trip, the less likelihood of disasters.

In addition to an individual patient's intrinsic factors, the experience (and all of the intrinsic factors) of the group forms a separate risk factor. On a wilderness trip, the group is only as strong as the weakest member, and the trip should be tailored to the weakest member not the most able. When doing a wilderness PPE, it is important to ask about the composition of the group. Will the patient be the leader of the group or one of the least experienced and weakest? Understanding the limitations of the individuals within and of the overall group is imperative because having to carry an individual for 
even a short distance has a major impact on a trip. For novices, it is also important to question the qualifications of the group leader as this can have a large effect on the success and safety of the trip.

In summary, the physician needs to do a thorough inventory of extrinsic and intrinsic factors and assess whether the limitations of the intrinsic factors are able to meet the demands of the extrinsic factors. Although errors from extrinsic factors usually result from underestimating these risks, rescues due to intrinsic factors frequently stem from overestimating one's skills. The combination of underestimating risk and overestimating skills is a common denominator in many wilderness rescues.

\section{Patient Identification}

One of the more difficult areas is to identify who needs a wilderness PPE. With sports PPEs, it is relatively clear that anyone who participates in organized sports generally needs a PPE and patients schedule appointments specifically for that reason. The situation is less clear with the wilderness examination. Does a wilderness PPE apply to a fit 25-year-old man going on a 3-mile hike in a local park or 50-year-old planning a 1-day Grand Canyon Rim-to-Rim hike? Clearly, there are risks inherent in either activity, and what they have in common is that neither would automatically schedule a wilderness PPE with a physician before embarking on their hikes unless they were triggered by concern for a particular health condition or by requirement from an event or adventure organizer. Given that limitation, physicians need to inquire of their patients during routine visits about their exercise habits and may discover wilderness activities that they engage in or plan to engage in.

As mentioned, the wilderness PPE encompasses many types of individuals and activities, and it is difficult to devise a single PPE for every type of experience. However, most wilderness PPEs can be divided into 2 types: general outdoor experiences and specific trips. The general experience is for patients who are doing an activity on a regular basis, such as weekly day hiking. These activities are ongoing and generally not part of an organized group and the patient engages in as part of their regular exercise. Physician advice in these cases is generally focuses on general fitness and any injuries that may occur. Patients may seek physician counsel before beginning these activities but do not routinely seek physician guidance for medical clearance. Although the risk of these types of activities may be low, they are not without injuries and still warrant evaluation.
It is more likely that physicians will see patients in anticipation of a specific trip. These are usually specific events and occasionally do require medical releases as discussed above. The level of exertion is likely to exceed what the patient usually experiences, may require skills that the patient does not have, and involves a greater amount of risk than more traditional sports. This is where the identification of extrinsic and intrinsic factors can be extremely valuable in ensuring a safe and enjoyable experience for the patient. The 2 types of situations are summarized in Table 4.

\section{Timing of Preparticipation Examination}

The optimal timing of the wilderness PPE depends to a large degree on the type of activity anticipated and the intrinsic factors of the patient. For general outdoor activities on a regular basis, there is no specific timing required. Patients should be advised to gradually increase their activity and about proper equipment. There are also some general considerations that apply to many wilderness activities, even short day trips. One of the most important, especially when going alone, is to alert someone as to the route and time of return. In cases of emergency, this can focus a search area and markedly reduce rescue time. Having a whistle is another essential, as cell phone service can be erratic even a mile from a road. The addition of extra water, paper and pen, and a flashlight or headlamp can also be useful in emergencies. Finally, carrying lightweight rain gear is important in most environments. Hypothermia (discussed elsewhere) can occur at surprisingly high temperatures once a person has gotten wet.

Although the physician may not be in a position to offer specific advice, there are many reputable shops or other trip-specific resources that offer excellent advice on equipment. For the specific trip, the more lead time before departure, the more likely the patient will be well prepared. For most activities, the author recommends at least 6 weeks of training depending on the baseline level of fitness and wilderness experience. The recommendation would be to simulate as closely as possible the

Table 4. Types of wilderness PPE

\begin{tabular}{lll}
\hline & Specific Trip & General Outdoor \\
\hline Trip length & Limited & Ongoing \\
Liability release & Usually needed & Not needed \\
Organized support & Usually & Rare \\
Environmental concerns & May be extreme & Usually low \\
Level of exertion & High & Usually low \\
Specific training & Usually needed & General fitness \\
\hline
\end{tabular}


environmental conditions to be encountered. This is not always possible (as in the case of extreme altitude), but creative training can prepare for many different conditions. Evaluation of patients 6 to 8 weeks in advance of an activity or adventure will also allow time for further diagnostics and testing if needed. It is the role of the physician to assess and find ways to reduce the discrepancy between the activity demands and the patient limitations. This may lead to situations where bridging that gap is not possible because of the extreme demands, patient factors, or inadequate time to correct the underlying problems. In this case, the physician must recommend not pursuing the planned experience or modifying it. This is especially a possibility when there is inadequate lead time. Unless the patient has a medical release form that needs to be signed by the physician, the advice can only be a recommendation for which the patient is free to ignore or follow. Although the well-being of the individual patient is the main concern of the physician, in these cases allowing an unqualified individual to participate in a wilderness group activity potentially reduces the enjoyment for everyone and jeopardizes the safety of the entire group.

\section{Qualification of the Examiners}

What are the qualifications for a physician to conduct the wilderness PPE? Just as it is not a requirement for a physician to have played a particular sport to do a sports PPE on a particular athlete, a physician does not need extensive wilderness experience to do a PPE. Most physicians do not have diabetes mellitus but are able to care for patients with this disease as long as they understand its pathophysiology and treatment. The same is true for the wilderness PPE. A primary care physician is well qualified to assess the intrinsic factors of his/her patients with emphasis on any underlying conditions that are being treated or medications that can interfere with activity. The major requirement to perform a wilderness PPE is the ability to ask thorough questions about the patient's planned activity and gain an understanding of the physical and environmental challenges. Once that is assessed, the physician can review the patient's medical history and physical examination to discover any limitations that would conflict with the anticipated demands. Ideally, all primary care physicians should be able to conduct this type of an examination, especially with access to resources such as the articles in this theme issue.

\section{Summary}

The ultimate goal of the wilderness PPE is to educate and encourage patients to participate in outdoor pursuits in a safe and healthy manner. There are many studies to support the value of outdoor experiences in both illness and for general mental and physical well-being throughout the life cycle. ${ }^{12,13}$ Thus, the goal of the wilderness PPE is not to discourage, scare, or disqualify patients from participating but rather to ensure that potential hazards are avoided, injuries and illnesses that may worsen with exposure are addressed appropriated, proper training and preparation takes place, and people can enjoy the outdoors in a safe manner. With a minimal amount of additional education, physicians can adequately assess both the intrinsic and extrinsic risk factors, address them and create a positive environment for their patients.

\section{References}

1. Department of Agriculture \& Forest Service. National Visitor Use Monitoring Results 2005-2009. http://www.fs. fed.us/recreation/programs/nvum/nvum_national_summary_ fy2009.pdf. Accessed May 29, 2014.

2. Kurowski K, Chandran S. The preparticipation athletic evaluation. Am Fam Physician. 2000;61(2683-2690): 2697-2698.

3. Schmied C, Borjesson M. Sudden cardiac death in athletes. J Intern Med. 2014;275:93-103.

4. Giese EA, O'Connor FG, Brennan FH, et al. The athletic preparticipation evaluation: cardiovascular assessment. Am Fam Physician. 2007;75:1008-1014.

5. AHA/ACSM Joint Statement, recommendations for cardiovascular screening, staffing, and emergency policies at health/fitness facilities. Med Sci Sports Exerc. 1998;30: 1009-1018.

6. Jette M, Sidney K, Blumchen G. Metabolic equivalents in exercise testing, exercise prescription and evaluation of functional capacity. Clin Cardiol. 1990;13:555-565.

7. Mcintosh SE, Leemon D, Visitacion J, et al. Medical incidents and evacuations on wilderness expeditions. Wilderness Environ Med. 2007;18:298-304.

8. Hung EK, Townes DA. Search and rescue in yosemite national park: a 10-year review. Wilderness Environ Med. 2007;18:111-116.

9. Mason RC, Suner S, Williams KA. An analysis of hiker preparedness: a survey of hiker habits in New Hampshire. Wilderness Environ Med. 2013;24:221-227.

10. Green GA, Winkler M, Cohen H. A prescription for wilderness safety. Your Patient and Fitness. 1993;7:17-21.

11. Krakauer J. Into Thin Air: A Personal Account of the Mt. Everest Disaster. New York: Anchor; 1999.

12. Puett R, Teas J, España-Romero V, et al. Physical activity: does environment make a difference for tension, stress, emotional outlook, and perceptions of health Status? J Phys Act Health. 2014;11:1503-1511.

13. Cedervall Y, Torres S, Aberg AC. Maintaining well-being and selfhood through physical activity: experiences of people with mild Alzheimer's disease. Aging Ment Health. 2014;30:1-10. 